\title{
Effectiveness of Cefoperazone-sulbactam alone and Combined with Tigecycline in the Treatment of Multi-drug Resistant Acinetobacter Baumannii Pulmonary Infection
}

\author{
Qin Lv1, Yanling Deng2, Xiaobo Zhu³, Jia Ruan ${ }^{4}$ and Lin Chen¹ \\ 1Department of Respiratory and Critical Care Medicine, Sichuan Academy of Medical Sciences \& Sichuan Province People's Hospital, China \\ 2Department of Internal Medicine, Cheng Du Aerospace Hospital, China \\ ${ }^{3}$ Department of Respiratory Diseases, Ziyang City People's Hospital, China \\ ${ }^{4}$ Department of Respiratory Diseases, Sichuan West China Hospital, Geriatric Center-Fifth People's Hospital of Sichuan Province, China
}

\begin{abstract}
The aim of this study was to compare the effectiveness of cefoperazone-sulbactam alone and combined with tigecycline in the treatment of multi-drug resistant acinetobacter baumannii pulmonary infection. It was an experimental study carried out from April 2016 to September 2018. One hundred and fourteen patients with multi-drug resistant acinetobacter baumannii pulmonary infection were randomly divided into group A and group B with 57 cases in each group. Group A was treated with cefoperazone-sulbactam sodium alone, and group B was treated with cefoperazone-sulbactam combined with tigecycline. After 14 days of treatment, serum levels of PCT, CRP, TNF- $\alpha$ and IL- 6 in group B were lower than those in group A (all $p<0.001$ ); APACHE II scores of group B were lower than those of group A ( $p<0.001)$. Compared with cefoperazone-sulbactam sodium alone, cefoperazone-sulbactam combined with tigecycline can effectively reduce the inflammatory response of patients with multi-drug resistant acinetobacter baumannii pulmonary infection; and thus a better prognosis can be obtained.
\end{abstract}

Key Words: Cefoperazone-sulbactam, Tigecycline, Multi-drug resistant, Acinetobacter baumannii, Pulmonary infection.

How to cite this article: Lv Q, Deng Y, Zhu X, Ruan J, Chen L. Effectiveness of cefoperazone-sulbactam alone and combined with tigecycline in the treatment of multi-drug resistant acinetobacter baumannii pulmonary infection. J Coll Physicians Surg Pak 2020; 30(3):332-334.

Acinetobacter baumannii is non-fermentative and aerobic gram-negative bacteria, which belongs to acinetobacter group. ${ }^{1}$ Because of the mild growth environment, acinetobacter baumannii is easy to multiply in large quantities, thus breaking the normal flora balance in the host body, leading to the immunity decline of the patients, and often causing infection. The most common organ affected by nosocomial infection caused by acinetobacter baumannii is lung. Drug resistance of this organism is becoming more and more serious, which leads to the limitation of clinical medication. Cefoperazone-sulbactam is the combined synergist of the third generation cephalosporin. Some studies have found that cefoperazone-sulbactam has bactericidal effect on acinetobacter. ${ }^{2}$ Tigecycline is a new type of glycylcycline antibiotic, which is not subject to the changes of $\beta$-lactamase, target modification, efflux pump, and enzyme target. It can antagonize the

Correspondence to: Dr. Lin Chen, Department of Respiratory and Critical Care Medicine, Sichuan Academy of Medical Sciences \& Sichuan Province People's Hospital, 610072, China E-mail:cgbrx77@163.com

Received: December 07, 2018; Revised: April 09, 2019; Accepted: July 09, 2019 ribosome drug resistance mechanism of acinetobacter baumannii and inhibit the bacteria to excrete the antibiotics. A study has shown that tigecycline is safe and effective in the treatment of multi-drug resistant bacteria in ICU. ${ }^{3}$ An in vitro antimicrobial experiment showed that tigecycline combined with cefoperazonesulbactam has synergistic effect and is a good method for the treatment of multi-drug resistant acinetobacter baumannii. 4

The aim of this study was to compare the effectiveness of cefoperazone-sulbactam alone or in combination with tigecycline in the treatment of multi-drug resistant acinetobacter baumannii pulmonary infection.

This study was carried out at the Department of Respiratory and Critical Care Medicine, Sichuan Academy of Medical Sciences and Sichuan Province People's Hospital, China, from April 2016 to September 2018. It was approved by the Hospital Ethics Committee. A total of 114 patients with multi-drug resistant acinetobacter baumannii pulmonary infection were selected as the research subjects. The inclusion criteria were that the patients were diagnosed as multi-drug resistant acinetobacter baumannii pneumonia by imaging examination and sputum culture. Before the use of antibiotics, the patients coughed up sputum, and samples were taken 
Table I: Comparison of therapeutic indices between the two groups.

\begin{tabular}{l|l|c|c|c}
\hline Indices & Time & $\begin{array}{c}\text { Group A } \\
(\mathrm{n}=57)\end{array}$ & $\begin{array}{c}\text { Group B } \\
(\mathrm{n}=57)\end{array}$ & -value \\
\hline PCT $(\mu \mathrm{g} / \mathrm{L})$ & Before treatment & $8.73 \pm 1.41$ & $8.69 \pm 1.63$ & 0.889 \\
& After 14 days of treatment & $3.46 \pm 0.82$ & $1.05 \pm 0.47$ & $<0.001$ \\
\hline $\mathrm{CRP}(\mu \mathrm{g} / \mathrm{L})$ & Before treatment & $395.21 \pm 21.74$ & $391.86 \pm 23.05$ & 0.426 \\
& After 14 days of treatment & $277.95 \pm 13.16$ & $191.73 \pm 10.28$ & $<0.001$ \\
\hline TNF- $\alpha(\mu \mathrm{g} / \mathrm{L})$ & Before treatment & $63.42 \pm 4.35$ & $63.65 \pm 5.02$ & 0.794 \\
& After 14 days of treatment & $39.74 \pm 3.09$ & $26.88 \pm 2.71$ & $<0.001$ \\
\hline IL-6 $(\mu \mathrm{g} / \mathrm{L})$ & Before treatment & $153.81 \pm 16.92$ & $154.03 \pm 17.44$ & 0.946 \\
& After 14 days of treatment & $83.92 \pm 8.17$ & $59.16 \pm 6.05$ & $<0.001$ \\
\hline APACHE II & Before treatment & $19.65 \pm 1.94$ & $19.78 \pm 1.66$ & 0.701 \\
& After 14 days of treatment & $14.06 \pm 1.33$ & $11.94 \pm 1.02$ & $<0.001$ \\
\hline
\end{tabular}

after gargling. The age of the patients was over 18 years old. The hospitalisation time of the patients was over 48 hours. Patients with pulmonary tuberculosis and immune diseases; pregnant or lactating women; with mental disorders who could not communicate normally; drug allergies used in this study; who had taken antibiotics in the near future; and patients who could not complete the treatment were excluded.

According to number table method, the patients were blinded and randomly divided into group A and group B with 57 cases in each group. After admission, the vital signs of the two groups were closely observed and the balances of water and electrolyte and acid-base were maintained. Group A was treated with cefoperazonesulbactam sodium alone, i.e. $3 \mathrm{~g}$ cefoperazone-sulbactam sodium was added into $100 \mathrm{~mL} 0.9 \%$ sodium chloride solution by intravenous drip, once every 8 hours.

Group B was treated with cefoperazone-sulbactam combined with tigecycline. For the first day, $100 \mathrm{mg}$ tigecycline was injected intravenously into $250 \mathrm{~mL} 0.9 \%$ sodium chloride solution. On the second day, $50 \mathrm{mg}$ tigecycline was added into $0.9 \%$ sodium chloride solution by intravenous drip, twice a day. Both groups were treated continuously for 14 days.

Before as well as 14 days after the treatment, $5 \mathrm{ml}$ venous blood was collected in the morning, centrifuged, and serum was separated. The levels of serum procalcitonin (PCT), C-reactive protein (CRP), tumor necrosis factor- $\alpha$ (TNF- $\alpha$ ) and interleukin-6 (IL-6) were detected by double antibody enzyme-linked immunosorbent assay. Acute physiological and chronic health status (APACHE II) score was used to evaluate the prognosis of patients. The higher the score was, the worse the prognosis would be.

SPSS 25.0 software was used for data analysis. Measurement data were expressed as mean \pm standard deviation. Independent sample t-test was used for comparison. The significance level was set at $p<0.05$.

Among the 114 patients, there were 60 males (52.63\%) and 54 females $(47.37 \%)$. The age ranged from 26 to 77 $(62.18 \pm 4.25)$ years. Twenty-four patients $(21.05 \%)$ had coronary heart disease and 13 patients $(11.40 \%)$ had diabetes mellitus. Before treatment, there was no significant difference in serum PCT, CRP, TNF- $\alpha$ and IL-6 levels between the two groups $(p=0.889,0.426,0.794$, $0.946)$. After 14 days of treatment, the serum levels of PCT, CRP, TNF- $\alpha$ and IL-6 in group B were lower than those in group $A$ (all $p<0.001$, Table I).

There was no significant difference in APACHE II score between the two groups before treatment $(p=0.701)$. After 14 days of treatment, APACHE II scores in group B were lower than those in group A $(p<0.001$, Table I).

Endotoxin and lipopolysaccharide released by acinetobacter baumannii after its invasion can stimulate mononuclear macrophages and neutrophils to release inflammatory factors, while the chemotaxis and activation of the latter can result in secretion of a large amount of inflammatory factors, thus causing systemic inflammatory response syndrome. CRP, TNF- $\alpha$ and IL-6 are common inflammatory factors clinically. CRP is an acute phase reactive protein synthesised by hepatocytes stimulated by inflammation such as pathogenic bacteria infection and tissue damage. It can activate complements and enhance the phagocytosis of leukocytes and mononuclear macrophages. TNF- $\alpha$ is an important proinflammatory factor, which can promote the activation of macrophages and B lymphocytes; and cause the injury of vascular endothelial cells. IL-6 is one of the most recognised important indicators for early diagnosis of bacterial infections. PCT can be used as a sensitive index to judge the existence and severity of infection.

One study confirmed that cefoperazone-sulbactam could improve antimicrobial activity of tigecycline against ventilator-associated pneumonia caused by extensively drug-resistant acinetobacter baumannii. ${ }^{5}$

This study showed that cefoperazone-sulbactam combined with tigecycline could reduce the serum levels of PCT, CRP, TNF- $\alpha$, IL-6 more effectively than cefoperazone-sulbactam sodium alone.

APACHE II score is an authoritative scoring method for evaluating the prognosis of critically ill patients. This study found that the APACHE II score of patients treated with cefoperazone-sulbactam combined with tigecycline was lower than that of patients treated with cefoperazonesulbactam sodium alone. It is suggested that cefoperazone-sulbactam combined with tigecycline may improve the prognosis of patients.

The clearance rate of bacteriology of cefoperazonesulbactam combined with tigacycline in the treatment of multiple-drug resistant acinetobacter baumannii pulmonary infection has not been observed. Thus, it needs further study in the future.

CONFLICT OF INTEREST:

Authors declared no conflict of interest.

\section{AUTHORS' CONTRIBUTION:}

QL: Data collection, compiling, statistics and discussion. 
YD: Literature review and data referral.

$X Z$ : Literature review and discussion.

JR: Supervision and revision.

LC: Supervision and final approval.

\section{REFERENCES}

1. Ramadan RA, Gebriel MG, Kadry HM, Mosallem A. Carbapenemresistant Acinetobacter baumannii and pseudo-monas aeruginosa: Characterization of carbapenemase genes and E-test evaluation of colistin-based combinations. Infect Drug Resist 2018; 11:1261-9.

2. Wieczorek $P$, Sacha $P$, Ojdana $D$, Milewski $R$, Jurczak $A$, Kaczynska $\mathrm{K}$, et al. In vitro resistance development in acinetobacter baumannii to sulbactam and cefoperazone. Med Dosw Mikrobiol 2012; 64:55-62.
3. Tumbarello M, Pennisi MA, Bello G, Antonelli M, Maviglia R, Bernini $\mathrm{V}$, et al. High dose tigecycline in critically ill patients with severe infections due to multidrug-resistant bacteria. Crit Care 2014; 18:1-9.

4. Liu $\mathrm{B}$, Bai $\mathrm{Y}$, Liu $\mathrm{Y}, \mathrm{Di} \mathrm{X}$, Zhang $\mathrm{X}$, Wang $\mathrm{R}$, et al. In vitro activity of tigecycline in combination with cefoperazonesulbactam against multidrug-resistant acinetobacter baumannii. J Chemother 2015; 27:271-6.

5. Qin Y, Zhang J, Wu L, Zhang D, Fu L, Xue X. Comparison of the treatment efficacy between tigecycline plus high-dose cefoperazone-sulbactam and tigecycline monotherapy against ventilator-associated pneumonia caused by extensively drugresistant acinetobacter baumannii. Int J Clin Pharmacol Ther 2018; 56:120-9. 